\title{
Modérer la langue politique à l'extrême
}

Les journalistes remarqueurs au début de la révolution française

Moderating political language to an extreme: journalistic commentators at the beginning of the French Revolution

Jacques Guilhaumou

\section{(2) OpenEdition \\ Journals}

\section{Édition électronique}

URL : https://journals.openedition.org/ahrf/10528

DOI : 10.4000/ahrf.10528

ISSN : 1952-403X

Éditeur :

Armand Colin, Société des études robespierristes

Édition imprimée

Date de publication : 1 septembre 2009

Pagination : $21-45$

ISBN : 978-2-200-92559-8

ISSN : 0003-4436

\section{Référence électronique}

Jacques Guilhaumou, " Modérer la langue politique à l'extrême », Annales historiques de la Révolution française [En ligne], 357 | juillet-septembre 2009, mis en ligne le 01 septembre 2012, consulté le 22 avril 2022. URL : http://journals.openedition.org/ahrf/10528 ; DOI : https://doi.org/10.4000/ahrf. 10528 
A R T I C L E S

\title{
MODÉRER LA LANGUE POLITIQUE À L'EXTRÊME. LES JOURNALISTES REMARQUEURS AU DÉBUT DE LA RÉVOLUTION FRANCAISE
}

Jacques GUILHAUMOU

\begin{abstract}
Nombre de journalistes patriotes, au début de la Révolution française, multiplient les remarques sur les nouveaux usages de la langue politique, certes pour en déplorer les abus - c'est le fameux thème au XVIII ${ }^{e}$ siècle d' " abus de mots "-, mais aussi pour s'interroger sur leur " signification précise ». Ces remarqueurs de la langue s'inscrivent dans une tradition linguistique ancienne, mais apportent, dans leur multiple " observation sur les mots " (Duquesnoy), des éléments précieux pour la connaissance de la langue politique pendant la Révolution française. Plutôt modérés politiquement, ils n'en abordent pas moins les usages de la langue dans leur principe extrême, c'est-à-dire dans l'exagération verbale, pour mieux opérer leur réduction analytique, et ainsi ne pas en laisser le bénéfice aux pamphlétaires royalistes.
\end{abstract}

Mots-clés : langue politique, remarques linguistiques, modération, exagération.

Dans une de ses nombreuses notes consacrées à la définition de mots tels que insurrection, peuple, monarchie etc., le journaliste patriote Adrien Duquesnoy écrit, dans L'Ami des patriotes : "Je prie qu'on me pardonne mes fréquentes observations sur les mots $»^{1}$. Dénoncer l'abus des mots, s'interroger sur l'acception de «quelques expressions nouvelles », s'évertuer d'employer tel ou tel mot dans sa « signification pré-

(1) Livraison du 11 décembre 1790. La plupart des sources citées, journaux et autres, proviennent de la BNF. Nous le précisons dans le cas contraire. 
cise », tel est le travail d'observation et d'analyse que pratiquent de très nombreux journalistes au début de la Révolution française ${ }^{2}$.

Cependant observer, analyser ne suffit pas à remarquer l'ensemble des mouvements de la langue politique. Encore faut-il en mesurer les effets, d'où un intérêt particulier pour " la langue des effets », en l'occurrence "la langue de l'exagération", selon les expressions du patriote Cérutti. Une langue qui n'est pas simple constat de l'exacerbation de l'abus des mots, mais que l'on peut réduire à un principe, en la raccordant à « la science sublime des effets ». Principe s'entend ici au sens de Montesquieu, « force qui entraîne tout », ressort de l'activité, mode de subjectivation qui met en mouvement ${ }^{3}$. Qui plus est, dans l'héritage médiéval, le principe conjoint le « sujet», premier terme d'une proposition accidentelle, modératrice relative à la santé de l'homme, sa vertu et sa science, donc là où il est considéré comme une chose, et « l'extrême » qui marque le terme de cette proposition et des choses qu'elle représente ${ }^{4}$.

Or tel est bien le mouvement de la langue politique, au début de la Révolution française, de s'instituer certes progressivement langue nationale, ici dans une série ininterrompue de remarques linguistiques modérant ses usages, mais dans le même temps d'être mue par un principe extrême, le fait même de l'exagération verbale, ce qui suppose d'opérer la réduction en art d'une telle force langagière ${ }^{5}$.

Rendre compte de ce double processus permet alors d'appréhender l'ensemble du mouvement de la langue dans l'intervention de journalistes patriotes favorables à la modération des mots, jusque dans le fait même d'en mesurer la force du principe dans leur exagération même. Si ces journalistes s'essayent à normer la nouvelle langue politique à l'encontre de l'abus des mots, ils cherchent aussi, dans une démarche critique, à

(2) Nous inscrivons donc notre étude dans la lignée de l'intérêt des historiens de la langue pour les remarqueurs, c'est-à-dire pour ces hommes de lettres qui, dès le XVI $\mathrm{e}^{\mathrm{e}}$ siècle, multiplient les remarques sur la langue et ses usages, sans prendre le point de vue du grammairien. Voir sur ce point Philippe CARON (dir.), Les remarqueurs sur la langue française du XVI siècle à nos jours, Rennes, Presses Universitaires de Rennes, 2004.

(3) Nous avons déjà précisé ce qu'il est d'un tel principe dans le langage révolutionnaire à propos du journaliste et grammairien patriote Antoine Tournon dans notre article « Antoine Tournon, un journaliste patriote à l'épreuve des principes », $A H R F, \mathrm{n}^{\circ} 351$, janvier-mars 2008, p.3-28.

(4) Nous précisons cet apport initial, dans la formation de la langue politique, en conclusion de l'article.

(5) La réduction en art, dans le domaine de la langue, consiste à mettre en œuvre la réduction des variations d'usage pour « rendre la langue purement française », c'est-à-dire mettre en règles le vernaculaire à partir de «façons de parler » reprises dans des descriptions raisonnées. Voir sur ce point, Francine MAZIERE, «Émergence de la langue française », Les Français en émergence, Milan, Galazzi et Molinari, 2006, p. 9-21. 
réduire analytiquement l'exagération verbale, pour en positiver la force et surtout ne pas en laisser le bénéfice des effets aux extrémistes de tous bords, principalement les pamphlétaires royalistes en 1790-1791.

\section{Instituer le français langue nationale : un objet social à part entière}

En première approche, le travail des journalistes remarqueurs s'apparente à un discours normatif hérité d'une part des leçons de l'art de la conversation et du bel esprit en pleine expansion du temps des Lumières, d'autre part repris de la recherche, à l'encontre de l'abus des mots, d'une norme du bon usage sur une base analytique, donc par recours à l'analogie.

L'ouvrage récent de Marie-Anne Paveau et Laurence Rosier fournit une description très précise du système de valeurs et des modalités de description linguistique qui sous-tendent une telle pratique métalinguistique jusqu'à nos jours ${ }^{6}$. Dans cette voie, il est possible de reconstituer le trajet, pour la période révolutionnaire, d'une sociolinguistique spontanée des nouveaux usages du discours politique au plan descriptif, normatif et idéologique sur la base des attitudes explicatives, appréciatives et normatives des journalistes. Il s'agit tout à la fois d'une norme objective, avec l'usage intensif des mots du politique, d'une norme subjective, forte du sentiment de la langue politique, et enfin d'une norme directement politique au titre de la promotion de la langue de la liberté.

Nous allons prendre en compte de telles partitions d'une pratique métalinguistique qui nous donne une intéressante vue d'ensemble sur le discours normatif des révolutionnaires ${ }^{7}$, tout en l'insérant dans un critère méthodologique plus large, ayant trait à un processus de schématisation plus général des pratiques linguistiques. Il s'agit d'un processus linguistique de schématisation qui procède d'un jugement réfléchissant sur la langue, c'est-à-dire d'un jugement qui relève d'une norme subjective, le sens commun, investi dans un espace intersubjectif où chaque soi communique avec d'autres sois. Qui plus est, il se trouve que Kant a pensé ce processus de schématisation dans le cas de la Révolution française, en l'associant à sa réflexion sur le jugement en histoire. Il y reconnaît trois

(6) La langue française. Passions et polémiques, Paris, Vuibert, 2008.

(7) Agnès Steuckardt mène, et en dialogue avec nous, une enquête approfondie en ce domaine. Voir en particulier sa récente réédition du Dictionnaire national et anecdotique de PierreNicolas Chantreau, accompagnée d'une longue et précieuse présentation, Limoges, Lanbert-Lucas, 2009. 
éléments suivants qui se succèdent de l'un à l'autre : "la conviction commune », « la manière de penser proprement subjective » qui se traduit dans une disposition subjective de l'esprit, enfin «l'expression qui convient », trois éléments dont nous allons considérer la dimension sémiologique $^{8}$. Ainsi, du constat de "la conviction commune de l'abus des mots » à la recherche de "l'expression qui convient », en passant par « une manière de se réapproprier les mots » et de les rendre conformes à une disposition morale du genre humain, il s'agit - au-delà de la description d'éléments d'un discours puriste très répétitif-, d'identifier « une forme de jugement sur la langue » qui contribue, au même titre que les grammaires et les dictionnaires de la période révolutionnaire, à la construction de l'objet social « langue nationale ».

\section{Un trajet dans la langue}

\section{La conviction commune de l'abus des mots}

Ce qui fait sens commun, "conviction commune» pour utiliser une expression courante à l'époque révolutionnaire, chez les journalistes remarqueurs de la Révolution, c'est bien sûr l'abus des mots tel que l'explicite une fois de plus Duquesnoy: "I'ai assez remarqué, dans le cours de cet ouvrage, combien on abuse des mots «peuple, nation, pouvoir $»^{9}$, citant ainsi une série synonymique très classique, avec «multitude ", et le plus souvent commentée, du dictionnaire des Synonymes françois de l'abbé Girard à la Feuille Villageoise:

«Synonyme. C'est un nom ou un verbe qui a la même acception qu'un autre nom ou un autre verbe. Nation, Peuple, multitude sont des mots employés quelque fois l'un pour l'autre, mais qui diffèrent dans le fond. Multitude signifie un assemblage d'hommes réunis dans une place. Peuple signifie un assemblage de citoyens formant un empire plus ou moins étendu. Nation signifie un assemblage de provinces, vivant sous les mêmes lois, et parlant la même langue. On dit une multitude ignorante, un peuple industrieux, une nation éclairée. Ou peut dire aussi, un grand homme est estimé de sa nation, admiré du peuple, et quelque fois persécuté par la multitude $»^{10}$.

(8) Nous avons montré ailleurs le lien étroit entre ce processus et l'éthique de l'émancipation chez Kant, qui recoupe donc la valeur émancipatrice de la langue politique pendant la Révolution française, La parole des sans. Les mouvement actuels à l'épreuve de la Révolution française, Lyon, ENS Éditions, 1998, p. 108 sq.

(9) L'Ami des patriotes, $1^{\text {er }}$ janvier 1791.

(10) Feuille Villageoise, 13 octobre 1791. 
Une brochure royaliste de 1790, qui s'intitule de fait L'Abus des mots, s'inquiète ainsi d'une série d' « expressions fastueuses dont on nous étourdit tous les jours ", en l'occurrence liberté, propriété, patriotisme, aristocratie, despotisme, regénération, constitution, gloire de la nation. ${ }^{11} \mathrm{Et}$ de même, en l'an III, « Jamais l'abus des mots ne fut si commun », précise également une brochure titrée Essai sur le patriotisme. Enfin, des rubriques de journaux s'intitulent « Sur l'abus des mots », en particulier au cours de l'année 1792, propice avec son événementiel chargé à un tel constat, par exemple dans L'Ami des Patriotes du 22 avril 1792, et dans Le Patriote français du 9 mai 1792. D'une remarque à l'autre, le champ sémantique de cette expression s'organise alors de façon diversifiée, mais en partie attendue pour qui connaît ce thème très répandu au siècle des Lumières ${ }^{12}$.

Le premier aspect abordé, et de façon réitérative, concerne l'usage abusif des mots en tant qu'instrument de domination des hommes :

Les hommes se mènent par les mots/

On conduit les hommes par des mots

Les hommes se prennent aux mots/Tous les partis abusent des mots pour tromper les hommes

C'est toujours avec les mots que les despotes et les prêtres ont conduit la masse du genre humain

Les mots

ont une si grande influence en temps de révolution déterminent le mode de la Révolution

L'abus des mots, un instrument à diviser les hommes

à gouverner toutes les classes à désigner, former l'opinion publique

Il en ressort alors un accent particulier sur « la fausse application » des mots tant dans l'usage commun que du fait de l'abstraction, sur l'emploi de mots vides de sens par toutes sortes de procédés linguistiques, de la confusion des dénominations dans l'abus du raisonnement et la synonymie mal employée à des mots accolés ensemble sans signification précise :

(11) Bibliothèque Historique de la ville de Paris, BHVP $8220 \mathrm{~N}^{\circ} 1$.

(12) Cf. Ulrich Ricken, " Réflexions du XVIII" siècle sur "l'abus des mots" ", Mots, 1982, V.4, n ${ }^{\circ} 1$, p. $29-45$. 


\section{L'abus des mots}

Commun, fatal au public, funeste aux individus produit

l'abus du raisonnement

un usage continuel de l'abstraction

synonymie

mots employés l'un pour l'autre

(nation, peuple, multitude)

mots accolés ensemble

(liberté, égalité, constitution) les fausses applications des mots

insignifiance des mots, mots vides

de sens

mots accusateurs

(patriote, fédéraliste, terroriste..)

mots des partis, confusion de dénominations

(enragés, patriotes, modérés)

Le discours du journaliste remarqueur se construit alors à partir de la série suivante d'inférences :

- Distinguer ce qui est commun entre les mots :

On remarque des différences d'expression/On voit désigner sous ces deux dénominations

Essayons, si possible, de donner des mots X une idée distincte, de distinguer ce qu'ils ont de commun, ce qui les sépare

- Exprimer la signification des mots :

On emploie trop souvent ces deux mots, sans faire attention au sens qu'ils représentent

Il faut employer chaque mot dans une signification précise

Les mots doivent exprimer précisément ce que l'on veut qu'ils signifient

On doit apprendre à tous la vraie signification des termes

- Expliquer l'acception des mots :

On ne doit pas être indifférent à l'acception que les mots reçoivent du hasard et qui en pervertit le sens primitif

On doit être attentif à l'acception que nous donnons aux expressions nouvelles

Il faut sans cesse expliquer les termes dont on se sert

Il faut que les mots aient dans la langue un sens clair, juste, analogique

Il faut rendre aux mots la pureté de leur signification primitive et vraie 
Cependant la dénonciation de l'abus des mots reste le fait aussi bien du démocrate que du royaliste comme le montre l'intervention de ce dernier dans un dialogue entre eux, au sein d'un journal intitulé Les devoirs de la seconde législature. Journal didactique, critique et polémique: «On éclaire le peuple? Dites qu'on lui apprend à abuser des mots, et à entorser ou estropier les définitions le plus solidement établies pour nourrir et entretenir une fausseté de raisonnement qui tourne à la ruine même du peuple $\rangle^{13}$. Il convient alors d'en appréhender une extension plus spécifiquement patriotique lorsqu'il s'agit de prendre en compte la manière d'être, de faire, de se comporter, de dire du spectateur face à une telle variation incontrôlée des dénominations.

\section{Une manière de penser, de s'approprier les mots}

Autant l'abus des mots est sans cesse constaté en matière d'usage des «mots accusateurs", et tout particulièrement le couple bien connu aristocrates/patriotes, ou plus largement des « dénominations des partis » propice à « la confusion de classe et de dénominations », autant là où une dénomination fait «ligne de démarcation » entre amis et ennemis de la liberté dans l'espace du on dit, il est d'abord question de ce qu'on se dit d'une telle dénomination, de ce qu'on entend par ce mot.

L'exemple en la matière le plus significatif est celui du mot « sansculotte ", à la fois par son parcours très particulier ${ }^{14}$ et par la manière dont il est repris, à diverses reprises, sous une forme dialogique d'échange, certes fictionnelle. Un « mot trivial», une « insipide plaisanterie » introduits dans la conversation par «l'insolente vanité de l'aristocratie», puis détournés « pour attirer au parti de quelques hommes tout l'intérêt qu'inspire la pauvreté », tels sont les termes qu'utilise Duquesnoy dans une longue rubrique au sujet de ce mot, sous le titre attendu d'Abus des mots. Si le mot sans-culotte se présente sous la forme d'une "plaisanterie légère, et d'assez mauvais goût », ce n'est pas principalement affaire de pauvreté, ajoute le modéré Duquesnoy, mais « c'est l'affection et la singerie de ceux qui s'abaissent au niveau du pauvre pour acquérir de l'élévation et des richesses ", écrit-il. Et il considère alors que ce mot devient injurieux du moment où les « soi-disant sans-culottes » se sont appropriés cette dénomination ${ }^{15}$.

(13) Tome V, cahier II du 7 mai 1791, p. 35.

(14) Cf. Annie Gefrroy, « Sans-culotte(s) (novembre 1790-juin 1792)», Dictionnaire des usages socio-politiques, fascicule 1, Paris, Klincsieck, 1985, p. 159-186.

(15) L'Ami des patriotes, 22 avril 1792. 
Reste que le mot sans-culotte s'inscrit, par une telle exposition au sentiment et à la souffrance sociale, certes ridiculisée, dans un habitus linguistique d'une ampleur bien plus large qu'une simple affaire d'abus des mots. L'habitus, selon le sociologue Pierre Bourdieu, est une manière particulière, mais constante d'entrer en relation avec le monde avec en son sein une part de connaissance à valeur anticipatrice ${ }^{16}$. Elle s'expose donc à la sensation, au sentiment, à la souffrance, comme c'est le cas présentement avec le mot sans-culotte, et en propose une forme de maîtrise, de gouvernement. Les penseurs thermidoriens, soucieux d'éradiquer le sans-culottisme, ne s'y tromperont pas. Ainsi de Jean-François de La Harpe dans Du Fanatisme dans la langue révolutionnaire lorsqu'il écrit: «Il fallait que la marque du civisme prononcé fût toujours d'insulter de toute manière au malheur, avec une brutalité qu'on ne peut qualifier qu'en rappelant que c'était celle du sans-culottisme : ce mot dit tout à des hommes que le sansculottisme a gouvernés si longtemps et veut gouverner encore $»^{17}$.

Le girondin Carra, dans les Annales patriotiques et littéraires, précise alors que le terme de sans-culotte, dans la mesure où les révolutionnaires se l'approprient, devient une désignation qu' « ils s'honorent tous également de porter ». Appropriation qui fait «ligne de démarcation » et donc fait débat en permanence, avec le bougé de cette ligne, sur ce que l'on entend par ce mot. Pour sa part, Carra considère qu'il s'agit d'amis de la liberté qu'ils soient riches ou pauvres :

« Voilà certainement un vrai sans-culotte, car ce titre ne consiste pas, comme on le croît vulgairement, à n'avoir pas de chausses. C'est l'insolente vanité de l'aristocratie qui a imaginé cette dénomination, et dès lors elle est devenue la ligne de démarcation entre elle et les amis de la liberté : c'est précisément parce qu'elle leur a donné cette désignation que, riches ou pauvres, ils s'honorent tous également de la porter.

On se dit sans-culotte pour signifier dans une langue connu des aristocrates qu'on n'est pas de leur bord, qu'on veut être rangé dans la classe nombreuse qui, lassée de leur impertinent orgueil et de leur longue oppression, s'est levée pour les terrasser, et a juré leur chute éternelle ${ }^{18}$.

(16) Méditations pascaliennes, Paris, Seuil, 1997, p. 170 sq.

(17) Du fanatisme dans la langue révolutionnaire ou de la persécution suscitée par les Barbares du dix-huitième siècle, contre la religion chrétienne et ses ministres, 1797, p. 133. Disponible sur Gallica.

(18) Annales patriotiques et littéraires, 25 août 1793. 
«On se dit sans-culotte », le terme se précise ainsi dans son processus même d'appropriation, d'où la multiplication de formulations du type «Qu'entendez-vous par sans-culotte?», ou « qu'entends-tu par sans-culotte ? », aussi bien dans les affiches ${ }^{19}$, ce qui marque d'autant l'importance de ce travail de réappropriation, que dans la presse. Questionnements qui nous valent des dialogues assez savoureux, par exemple le suivant sur une affiche :

« Mais... qu'est-ce qu'un SANS-CULOTTE?

$\mathrm{Ah}$ ! ah ! maître fripon, lui dit alors un freluquet à culottes étroites, dis moi, QU'ENTENDS-TU par SANSCULOTTE, ou je t'assomme?

Un sans-culotte... un sansculotte... eh bien! puisqu'il faut que je vous le dise : un sans-culotte d'aujourd'hui est un sansculotte qui a de bonnes culottes, et cependant qui veut attraper les culottes de ceux qui ont des culottes, pour ne donner ni sou, ni maille ni culottes aux pauvres diables de sansculottes ».

Ainsi la définition du sans-culotte et du sans-culottisme s'avère d'une grande instabilité sémantique. À la question réitérative sous des formes telles que « Qu'entendez-vous par des sans-culottes ?», «Qu'entends-tu par sans-culotte ? » ou au constat que « ce mot de sans-culottisme n'est pas encore bien entendu », la réponse est hésitante, «j'entends, j'entends... », «Un sans-culotte, un sans-culotte... eh bien ! ». De même il s'y s'adjoint un commentaire métadiscursif, du type " puisqu'il faut que je vous le dise », « Mais vous me faites une question ridicule : tout le monde sait ce qu'on entend par sans-culotte », qui nous introduit soit à des différenciations sociales : «j’entends les non-propriétaires, les gens qui n'ont rien », - qui relève plus, dialogue oblige, du on dit que de la vérité sociale -, soit à un simple constat d'existence, « un sans-culotte d'aujourd'hui... », certes de forte teneur idéologique.

Reste toujours l'attestation d'un effort, au sein de la presse jacobine, pour trouver ce qu'il en est de « la vraie signification du mot », en le situant du côté de la vertu sociale, donc une fois de plus hors du clivage entre le riche et le pauvre :

(19) Ainsi dans les deux affiches issues de l'imprimerie de Gorsas, donc des jacobins brissotins en 1792, et conservées à la Bibliothèque de l'Assemblée nationale, sous le titre Un freluquet... aux pauvres b... de sans-culottes qui n'ont que des chaises de paille et Mais... qu'est-ce qu'un sans-culotte? 
«Quant au sans-culottisme, nous croyons que ce mot n'est pas encore bien entendu dans la plupart des Départements, où l'on a cherché à égarer le peuple. Les aristocrates qui, avec raison, regardent les Sans-Culottes comme leurs plus cruels ennemis, les ont dépeints comme des brigands, des anarchistes qui ne respectent rien, ni les lois, ni les propriétés, des hommes de sang qu'il faut exterminer.

Cependant les Sans-culottes ne sont moins rien que tout cela. Le vrai sans-culotte est l'image de la vertu sociale, il peut exister chez le riche comme chez le pauvre : c'est une réunion d'hommes, jaloux de la Liberté et de l'Égalité, ne respirant, que pour la gloire, l'indépendance et le bonheur de leur patrie, toujours prêts à se sacrifier pour le salut de leurs frères, qui n'estiment que les hommes qu'autant qu'ils sont vertueux et utiles à leurs semblables $»^{20}$.

L'art de raisonner à l'encontre de l'abus des mots procède ici de la façon suivante : c'est la manière même dont les sans-culottes eux-mêmes, spectateurs de l'usage de cette dénomination utilisée par les aristocrates comme une injure, en font une manière active de penser publiquement en la rapportant à un idéal moral qui lui confère une disposition d'esprit propice à l'émancipation humaine. À ce qui fait sens commun, l'abus des mots, succède donc une manière de déterminer une dénomination conforme à la disposition morale de l'humanité dans l'art même de trouver un mot propre. Reste que l'instabilité sémantique de sans-culotte, et son fort potentiel émotionnel, rendent ce terme peu apte à trouver l'expression qui convient. Il nous faut alors, dans la troisième étape du processus de schématisation, nous tourner vers un autre terme issu d'une considération sur un terme adversitif, « roi ».

\section{La quête de l'expression qui convient}

Nous entrons maintenant dans l'univers extensif de la recherche de l'expression qui convient. Elle relève très classiquement au XVIII ${ }^{\mathrm{e}}$ siècle de l'exercice analogique qui présentement permet de faire le partage entre « un mot vide de sens », « un mot vain et ridicule $»^{21}$ et un mot dont la signification est claire et précise, ce qui revient à rechercher «la vraie signification des termes $»^{22}$.

(20) Journal des Côtes de Cherbourg du 4 septembre 1793.

(21) Expressions du Mercure National et étranger , 30 mai 1791 et 18 février 1791.

(22) Expression de L'Ami des patriotes, 26 mars 1791. 
Une «Lettre sur le nouveau langage » publiée par un auteur anonyme dans la Correspondance patriotique du 14 novembre 1791 pose les jalons d'une telle démarche analogique, tout en se focalisant sur un exemple particulièrement parlant, celui du mot roi, et plus largement des termes qui s'y associent :

« Notre langue s'est élevée et devait s'élever. Elle a dû adopter et créer des mots nouveaux en recevant des idées nouvelles. Mais il aurait été bon que ces mots eussent dans la langue un sens clair, juste, analogique. Il est encore plus nécessaire de n'en pas adopter d'autres qui feraient contresens manifeste avec l'idée qu'on y attache. J'ai beaucoup de peine à m'accoutumer à l'expression liste civile, quand on parle du revenu, de la pension, $\mathrm{du}$ traitement que la nation assure au roi. Mais $[\ldots]$ quoique l'expression dont nous venons de parler manque de justesse, et ne soit pas conforme à la philosophie de la langue, l'usage fait qu'on la comprend ».

Pourquoi une telle réticence sur l'expression «liste civile», en quoi ne convient-elle pas? Sans doute parce qu'elle est associée aux manœuvres de ceux, autour du roi, qui veulent « royaliser l'esprit public » selon le journaliste du Courrier des départements ${ }^{23}$ à l'encontre donc de l'opinion publique. Ce dernier attribue ainsi à la «ménagerie de la liste civile » l'expression de « représentant héréditaire du genre humain » dans une rubrique intitulée une fois de plus «Sur l'abus d'un mot».

Le mot roi, dans une telle démarche analogique, fait d'autant plus problème «qu'on a cherché à le déguiser sous toutes les dénominations possibles ». Ainsi, « d'abord c'était le Chef suprême ; mais bientôt on en a fait un Fonctionnaire public, puis un Délégué !... Puis l'Agent de la Nation, puis le simple Électeur des Agents du pouvoir exécutif, puis enfin le Représentant ». Et pourtant : «Qui n'étant qu'un contre douze cents n'est pas plus que chacun des douze cents et n'a pas même sa voix contre eux tous ! » ajoute, non sans quelque ironie, le journaliste du Stationnaire au frontières, ou l'appel au bon sens ${ }^{24}$.

C'est aussi un mot qui permet au journaliste remarqueur de montrer sa capacité à rendre compte de "différences d'expression», en faisant attention au sens que les mots représentent, et donc à proposer « la vraie signification des termes ». Soit l'exemple suivant qui porte sur la néces-

(23) Dans sa livraison du 22 juillet 1792.

(24) Dans sa livraison du 28 août 1791. 
saire « différence d'expression » entre les mots « roi » et « pouvoir exécutif » :

« En examinant de près le style de la rédaction de l'acte constitutionnel, on remarque des différences d'expression qui en supposent dans le sens. Par exemple, tantôt on emploie le mot roi et tantôt le mot pouvoir exécutif; on voit évidemment que les comités ont voulu, sous ces deux dénominations, désigner deux autorités, deux pouvoirs distincts, de manière qu'il y ait les fonctions royales et les fonctions du pouvoir exécutif $»^{25}$.

Enfin les penseurs républicains s'emparent du mot roi pour le démonétiser complètement. Tout d'abord, ils opposent au Roi, ramené à une simple interpellation par son prénom, Louis, l'expression "peuple roi $»^{26}$. Puis ils en viennent, sous la plume de François Robert, journaliste mais par ailleurs auteur d'un fameux opuscule sur les Avantages de la fuite de Louis XVI et nécessité d'un nouveau gouvernement ${ }^{27}$, à demander la suppression du « funeste nom de roi » puisqu' « un roi n'est rien » selon la règle même de l'analogie entre les mots et les choses. Et il s'agit aussi d'en finir avec toutes les «expressions indécentes » qui s'y associent, telles que « ses fidèles sujets » et « la liste civile». " Protester contre la dénomination de sujet », « Désigner la liste civile comme le simple salaire du roi », tel est ici le propre d'une démarche analogique qui met en évidence « un rapport secret par lequel deux objets se conviennent $»^{28}$.

Le journaliste en vient enfin à remarquer, - toujours dans l'environnement de roi, mais dans l'ordre adjectival -, la différence entre royal et «national », qui nous introduit, par la substitution de la « nation » au « roi », à ce qu'il appelle « un synonyme de remplacement » :

«Plusieurs auteurs aussi savants qu'ingénieux ont pris plaisir à nous faire sentir la délicatesse de notre langue, en nous prouvant qu'il n'est aucun mot qui puisse s'employer indifféremment à la place d'un autre. Ils auraient dû ajouter que non seulement un mot n'est pas le synonyme d'un autre, mais encore qu'avec le temps le même mot change souvent de

(25) Thermomètre du jour, 18 août 1791.

(26) Dans un texte anonyme fort intéressant de 1790 intitulé Sur l'influence des mots et le pouvoir de l'usage, et qui a fait l'objet, sous notre direction, d'une publication et d'un débat entre linguistes dans la seconde série $\mathrm{n}^{\circ} 1$ d'Archives et documents de la SHESL de décembre 1989.

(27) 1791, BNF $8^{\circ}$ Lb39 (5103), repris de Le républicanisme adapté à la France, 1790, disponible sur Gallica.

(28) Voir la rubrique « Analogie » dans la Feuille Villageoise, 13 octobre 1791. 
signification. Cependant, monsieur, comme les hommes ont toujours à peu près les mêmes idées à exprimer, il arrive alors qu'un mot, soit nouveau, soit ancien, prend la place de celui qui se trouve dépossédé. Ainsi, par exemple, la nation s'étant mise à la place du roi, le mot national a remplacé le mot royal. Mais il en est de ces synonymes de remplacement, comme des autres : ils ne sont jamais tout à fait justes. Je vais tâcher de faire sentir aux gens de goût les nuances délicates qui distinguent l'ancien royal du nouveau national $»^{29}$.

Nous pouvons donc considérer que les journalistes de la Révolution française, en multipliant leurs remarques sur les nouveaux usages de la langue politique, construisent une sorte de Dictionnaire contextuel des mots, comme nous l'avons déjà souligné ailleurs ${ }^{30}$, avec présentement une réflexion terminale sur l'abus de la synonymie, ainsi des mots aristocrate, révolte, propriétaire devenus respectivement synonymes de richesse, patriotisme et fripon. Cependant, nous risquons de manquer une dimension essentielle du processus linguistique ainsi mis en place, avec sa part évidente de modération analytique, si nous ne prenons pas en compte ce qui est, non pas son inverse, mais son complément analytique, l'exagération réduite à son principe, sa force propre.

\section{Un dictionnaire à la mode : la part de l'exagération}

Le Consolateur propose des éléments disparates, sous la plume de son rédacteur, d'un Dictionnaire à la mode, dont il justifie la nécessité du fait qu' " il est certain que beaucoup de mots Français ont changé d'acception et ne signifient plus la même chose $»^{31}$. Il considère en particulier tout « néologisme » dont « il faut s'habituer » et qui relève de l'invention de « termes génériques ». Ainsi l'art de dénoncer deviendrait la «fauchetterie », l'art de passer pour ce qu'on n'est pas, la « patrioterie ».

S'il est bien question de faire « appel au bon sens », comme le précise le rédacteur anonyme de Tirez le Rideau. La Farce est jouée (octobre 1791), pour, face à l'abus des mots, « s'entendre définitivement sur la vraie signification des mots mystérieux à l'aide desquels on a su

(29) Correspondance des mécontents, 8 avril 1791.

(30) «Fragments d'un dictionnaire contextuel des mots de la Révolution française », Cahiers de lexicologie ${ }^{\circ} 84,2004-1$, p. 119-134.

(31) $\mathrm{N}^{\circ} 24,23$ mars 1792. 
répandre ces illusions et tromper si cruellement la multitude », la tonalité est ici plutôt à la gaieté, voire à l'exagération.

De même, La Greffe patriotique de la société des amis du bon sens après avoir récusé tout ornement associé à la raison, se donne désormais en 1791 le droit de " planer au dessus de l'atmosphère pour voir s'il y a dans cette région quelque vérité politique » et donc de considérer que :

«La société admettra dans ses assemblées tous les déclamateurs qui s'y présenteront, mais sous la condition expresse que leurs phrases emphatiques, leurs locutions nouvelles, leurs exclamations, leurs exagérations outrées, leurs mouvements extatiques, leurs longues énumérations, en un mot tous leurs tours de force de quelque genre qu'ils soient, ne pourront servir à masquer aux yeux de la société, l'erreur, l'absurdité ou le mensonge, pour les introduire furtivement dans son sein ${ }^{32}$.

Nous entrons ainsi dans un espace dictionnairique particulier, au titre de l'exagération, dont nous n'avons conservé que des fragments, avec cependant une présentation d'ensemble de ce qu'aurait pu être un dictionnaire systématique. Nous la devons au patriote Cérutti, auteur par ailleurs de la Feuille villageoise, dans un texte imprimé de 56 pages, signé Micromegas, et intitulé Prospectus d'un Dictionnaire d'exagération ${ }^{33}$. Antoine de Baecque a bien décrit ce personnage si typique de la gaieté française qui se propose d'orner la langue française de ces « figures propres à convaincre les esprits les plus bornés $»^{34}$. S'il convient selon Cérutti de récuser tout usage du grossier, du grotesque, il importe tout autant de considérer l'exagération langagière, non avec les armes de la raison, donc de la bannir, mais de la disséquer jusque dans la parodie, de la réduire à ses principaux aspects, sur la base d'un principe définitoire d'elle-même, «l'excès de la force ». Avec une telle « attaque en parodie », s'occuper de l'exagération devient une activité en soi, comme il le précise dans la perspective de l'établissement d'un « Dictionnaire portatif et raisonné de tous les termes et de toutes les phrases qui forment la langue de l'exagération $»^{35}$.

(32) Ce curieux document se trouve dans le fonds des imprimés de la Collection Rondonneau, aux Archives nationales (AD I 65), p. 8.

(33) Disponible sur Gallica dans un recueil de quatre de ses textes des années 1780 et 1790.

(34) Dans le quatrième chapitre de son ouvrage, Les éclats du rire. La culture des rieurs au XVIII ${ }^{e}$ siècle, Paris, Calmann-Lévy, 2000.

(35) Prospectus... op.cit., p. 36. 
Le Dictionnaire à la mode devient ainsi l'espace où il s'agit, en parallèle avec la recherche analytique de "définitions exactes", de prendre en compte l'exagération en tant qu' " excès de la force », c'est-àdire dans son principe, ce qui nous fait entrer dans « la science sublime des effets », « la langue comme effet », par un travail de réduction en art. Alors qu' « il était temps d'enseigner un idiome proportionné à l'accroissement successif, ou plutôt à la crue subite et prodigieuse de nos idées », du fait que « c'est lorsqu'une science est perfectionnée qu'il convient de fixer la langue $\gg^{36}$, le choix d'une approche de l'exagération verbale peut étonner. Pourtant elle est le complément indispensable de la quête de la modération linguistique.

Il nous faut revenir ici au Rousseau penseur de la force des signes, en complément du Condillac principal théoricien de «la langue bien faite ». Dans la perspective rousseauiste ${ }^{37}$, définir les choses par leurs vrais noms, donc par des mots adéquats relève de l'identification de leur vraie forme, ce qui revient à prendre en compte la force des mots par le fait de la voix, l'imitation, la passion, bref ce qui fait impression dans le discours. Plus la présentation du sens est dramatique, plus l'accent est mis sur la force des signes, mieux l'on peut appréhender l'expressivité de la langue ainsi figurée. Et Cérutti de parler de «pantomime de l'exagération » et d'énumérer, à l'identique des Amis du bon sens, de tels effets extrêmes de la langue naturelle: "Exclamations puissantes, paroles énergiques, tournures inattendues, métaphores audacieuses, phrases de création $\gg^{38}$. Il s'agit alors de doubler, d'accentuer les mots, de les augmenter de volume. Ainsi un titre ridicule devient ridiculissime.

Il propose alors une "échelle de l'exagération» longue de dix barreaux, consécutive à la partie grammaticale supposée de son Dictionnaire $^{39}$. Il le fait au titre de «la partie philosophique» de son exposé, seule apte à rendre compte du « choix des termes et des phrases » par le déploiement d'un art particulièrement subtil. Il énumère ainsi, par l'art de la réduction, les formes de l'idiome exagérateur auxquelles nous allons donner un contenu lexical par la collecte d'éléments discursifs dans les nombreuses remarques des journalistes sur «les disputes de mots» qui

(36) Ibid., p. 38.

(37) Voir sur ce point l'ouvrage de Francine Markovits, L'ordre des échanges. Philosophie de l'économie et économie du discours au XVIIIe siècle en France, Paris, PUF, 1986, en particulier le chapitre II intitulé « Rousseau : l'efficace des signes ».

(38) Prospectus, op. cit., p. 40.

(39) Ibid., p. 44-53. 
constituent toujours l'élément principal de notre corpus d'analyse dans la présente étude.

1. L'emploi de termes aériens, donc situés dans « la partie la plus élevée de notre langue ». Et d'énumérer : nature, liberté, despotisme, fanatisme, enthousiasme, démocratie, etc.

Liberté, associée à nature, est bien sûr ici le maître-mot en la matière. Sous la plume des royalistes, elle est « esclavage horrible pour tous les honnêtes gens, mépris des lois et de toutes les conventions, etc... $»^{40}$. Elle conserve même son pluriel « libertés » d'Ancien Régime contre «nos prêcheurs de liberté » qui «menacent de l'infernale lanterne » toute personne prenant « le danger d'avoir une opinion contraire à la démence du moment ». Telle est la part de l'exagération, au regard de « la liberté raisonnable », de ce point de vue.

Cependant, à l'inverse, le patriote modéré considère que ce mot " désigne ce droit précieux que la nature accorde à tous les hommes", non sans une allusion à ce qui lui fait obstacle en ajoutant « [...] quand nature ne trouve point sur son chemin des bastilles qui ont des de Launay $»^{41}$, allusion malgré tout à la liberté du «cannibale isolé »-De Launay est massacré le 14 juillet 1789, jour même de la prise de la Bastille - propre à l'exagération du démagogue. Qui plus est, l'avènement de la liberté resémantise la série synonymique classique, nation, peuple, multitude, nous ouvrant l'esprit à la seconde étape sur l'échelle de l'exagération, de l'aérien au collectif.

2. L'usage des termes collectifs qui « servent à généraliser notre langue ", à l'exemple de la nation devenue une peuplade, le genre humain pris pour l'Univers.

Cette part de l'idiome exagérateur est admise même parmi les « grammairiens patriotes ». Ainsi Urbain Domergue, dans une rubrique intitulée « Du pouvoir des grands mots », considère qu' " il n'est pas vrai que certains mots ronflants, dont l'orateur remplit le vide de ses périodes, nuisent à l'effet qu'il doit produire, quoiqu'ils ne soient pas essentiels au fond du sujet $»^{42}$. Et de donner l'exemple de Cicéron parlant devant le peuple, de populi romani majestatem pour capter l'attention d'un peuple distrait.

Alors que le royaliste dénonce l'appel généralisant à « la gloire de la nation » qui couvre en fait les crimes du peuple parisien, la «barbare

(40) Synonymes nouveaux (1790), BHVP 7836.

(41) Dictionnaire national et anecdotique, op. cit., p. 158.

(42) Journal de Langue Française, tome 2, p. 459. 
joie » d'une « populace effrénée $»^{43}$, le patriote modéré n'en est pas moins critique : il considère que « nation a signifié tout parmi nous, dès l'instant que nous avons été réellement une nation $\gg{ }^{44}$, mais il retient le moment où le citoyen peut enfin crier «Vive la nation » après le 14 juillet 1789.

Faut-il considérer alors que « la nation s'étant mise à la place du roi, le mot national a remplacé le mot royal »? Nous entrons alors dans l'univers déjà mentionné des « synonymes de remplacement » et le journaliste de la Correspondance des mécontents d'ajouter : « Je vais tâcher de faire sentir aux gens de goût les nuances délicates qui distingue 1'ancien royal du nouveau national $\rangle^{45}$. Mais, dans le vocabulaire de l'exagération, la délicatesse n'est guère de mise.

3. L'usage de synonymes pour substituer à un terme délicat un terme véhément, par exemple « homme vendu ou à vendre au lieu d'un patriote impartial $»$.

Ainsi de même « le mot révolte est devenu synonyme du beau nom de patriotisme », et « puisque le changement d'un mot influe sur la chose publique », «le patriotisme a dégénéré en scélératesse. Le bandit, le voleur, l'assassin, l'homme jusqu'alors dévoué au mépris public, s'il porte une cocarde aux trois couleurs, est appelé patriote $»^{46}$.

Sur l'autre bord du couple patriote/aristocrate, un aristocrate, de « citoyen qui possède une dignité, une distinction, une charge honorable ou lucrative $»{ }^{47}$ devient $«$ le synonyme de mauvais citoyen, de pire encore : il devient un fauteur de complots, un ennemi de la liberté $»^{48}$. Et l'exagération porte alors sur le fait que «c'est le haro qui ordonne, oblige, qui force tout bon François à courir sus, à s'emparer de l'individu quelconque ou taxé d'aristocratie ». Les couples aristocrate/patriote, aristocrate/ démocrate se trouvent au centre d'un tel travail synonymique au début de la Révolution française. Mais une fois que richesse est devenue synonyme d'aristocrate, un nouveau couple, encore plus véhément, exagéré, se met en place aristocrate/sans-culotte.

Face à une telle exagération qui crée « une confusion de classes et dénominations », il s'agit de distinguer, dans les appellations de partis,

(43) L'Abus des mots, op. cit.

(44) Dictionnaire national et anecdotique, op. cit., p. 163.

(45) Correspondance des mécontents, 8 avril 1791.

(46) Ibid.

(47) Synonymes nouveaux, op. cit., p. 4.

(48) Dictionnaire national et anecdotique, op. cit., p. 108. 
telles que patriotes, modérés, enragés « ce qu'ils ont de commun, et ce qui les sépare $»^{49}$.

4. L'emploi d'images frappantes, de tournures vigoureuses à l'exemple d'un écrivain qui « dit que Dieu créa les Républiques dans sa bonté et les Monarchies dans sa colère ».

Ainsi en est-il lorsque le journaliste de $\grave{A}$ deux liards... mon journal écrit : «Avec le mot Fédéraliste par exemple, on a tué d'excellents républicains ; avec le mot Patriote, on a presque tué la République $»^{50}$. Mais c'est aussi le cas dans le sens donné au mot révolution lorsque "vous entendez dire des hommes qui abusent de ce mot, il faut des lois, des mesures, des hommes révolutionnaires $»^{51}$, propos appuyé par Condorcet dans le Journal d'Instruction sociale de juin 1793, tout en ajoutant «Entend-on par mesure révolutionnaire une mesure violente, extraordinaire, contraire aux règles de l'ordre commun? $\gg^{52}$ À vrai dire, c'est un terrain sur lequel l'abord de l'exagération est particulièrement intéressant, car sujet à hypothèse. En effet faut-il "justifier tous les excès en les rejetant » précise Condorcet, ce qui veut dire qu'il convient d'asservir à des règles même le plus excessif, en l'occurrence les lois révolutionnaires, ce qui permet d'en envisager l'hypothèse, par exemple dans le cas du maximum en matière de liberté du commerce des subsistances ${ }^{53}$. Une telle prise de conscience du danger à rejeter l'exagération sans donc en envisager l'hypothèse de la régulation analytique est l'élément central du Dictionnaire de l'exagération dont nous parcourons présentement les gradations.

Derrière la volonté affichée de trouver le mot juste, on peut aussi trouver quelque image frappante. Ainsi lorsque Urbain Domergue, recherchant la dénomination politique adéquate du nouvel État en 1791 propose celle de «loyaume », quelque peu ridicule à vrai dire, précise le journaliste du Bulletin ou Journal des Journaux ${ }^{54}$, il veut frapper l'imagination de son lecteur par ce qu'il croit être un néologisme de sa part. En fait le terme est déjà attesté en 1789. Il s'agit d'une brochure intitulée $L a$ trompette du jugement. Premier septembre 1789 où l'auteur anonyme

(49) Le Patriote Français, 10 mai 1792.

(50) Dans sa livraison du $\mathrm{n}^{\circ} 8,1792$.

(51) BrIssot, rubrique « Sur le mot révolutionnaire », dans Le Patriote Français, 21 avril 1792.

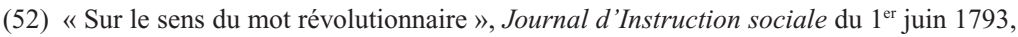
p. 4, Paris, Reprint Edhis, 1981.

(53) Ibid., p. 9.

(54) Dans sa livraison du 15 août 1791. 
pose la question, « Quel gouvernement faut-il donner à la France ? » et, à propos de la loi, répond: «La loi faite et acceptée par tous est notre unique souverain; si on pouvait employer ce mot sans heurter la grammaire, il faudrait dire, la France est un «loyaume », ou si on trouve plus auguste d'y mettre un mot grec, ce sera le mot de Nomarchie, de Nomos, qui veut dire loi $\rangle^{55}$. Nous sommes ici déjà dans la gradation suivante. colosse.

5. «Franchir toute gradation», ainsi faire de la grandeur un

C'est surtout ici, nous semble-t-il, le passage du tout au rien, et inversement. Ainsi des privilégiés, de la noblesse, et inversement du peuple. Là où la modération considère que la noblesse n'est rien « si vous entendez une qualité occulte, inhérente au sang », elle demeure malgré tout quelque chose « si vous entendez par ce mot talents, mérite, vertu, services ». L'exagération considère, pour sa part, qu'elle n'est vraiment rien. De même pour le roi : une fois que le peuple est « convaincu que les rois ne sont plus les maîtres », « il aura senti qu'un roi n'est rien », et donc qu'il est tout, qu'il est «peuple roi $»^{56}$. Il est aussi question ici de la gradation jusqu'à l'usure extrême de désignants déjà évoqués. Ainsi si l'on va « depuis le mot Aristocrate, qui commence à s'user, jusqu'au mot Terroriste, si à la mode aujourd'hui », « on peut compter dix adjectifs français ou francisés » dont l'abus est « fatal au bien public », précise le journaliste, là encore sensible au Dictionnaire à la mode ${ }^{57}$.

6. L'usage de comparaison qui flatte l'amour-propre, ainsi « d'attacher en quelque sorte un homme immortel à un homme vivant ».

Nous prendrons ici un exemple atypique, le cas de la mort de Mirabeau, comparé à Démosthène, à propos de son éloge funèbre dans La légende dorée ou les actes de martyrs pour servir de pendant aux actes des apôtres. Le rédacteur montre en quoi cette comparaison, d'exagérée devient insuffisante, dès le lendemain de la mort d'une personne. L'auteur de cet éloge d'écrire en effet : « Je l'ai souvent entendu appeler le Démosthène français ; mais, j'ose le dire, cette comparaison n'a rien de flatteur que pour l'orateur grec, et Mirabeau, dans des circonstances plus difficiles, déploya plus de talents et d'énergie $\gg^{58}$. Ce qui s'avère très égocentré du vivant de la personne prend une toute autre tournure après sa mort.

(55) Bibliothèque de l'Institut, GX 199 (21), p. 24.

(56) D'après François RoBert dans Avantage de la fuite de Louis XVI, op. cit.

(57) À deux liards...mon journal, $\mathrm{n}^{\circ} 8,1792$.

(58) La Légende dorée... 6 avril 1791, p. 218. 
7. « Renchérir sur ce qui se dit de plus fort».

Ce renchérissement est particulièrement visible, une fois de plus, dans l'usage gradué des désignants socio-politiques. Ainsi la Chronique de Paris précise les gradations par lesquelles les royalistes s'évertuent à rendre odieux les patriotes auprès de l'opinion publique ${ }^{59}$. «Tout l'art se réduit à trouver un nom », toujours plus fort que le précédent donc. D'abord «le mot jacobin a été habilement employé », alors que "les populaciers faisaient un usage non moins louable de celui d'aristocrate ", allusion sans doute principalement au Père Duchesne. « Maintenant, celui qui est à l'ordre du jour, c'est celui de factieux ». Face à une telle surenchère, le journaliste réagit en concluant : « Pour nous qui n'avons pas la même fécondité d'imagination, nous ne connaissons qu'un mot, un mot propre, dont l'équivalent est dans toutes les langues parce que la chose a été chez tous les peuples, c'est celui d'intrigant». Voilà une montée en généralité d'un désignant que l'on retrouvera dans d'autres conjonctures politiques, par exemple le fédéralisme de $1793^{60}$.

8. «S'approprier les termes les plus savants d'un art», dire ainsi des esprits qu'ils sont en incandescence, et non en effervescence.

C'est ici qu' " on dit » apparaît comme un terme usuel pour introduire l'avis de l'opinion publique ${ }^{61}$. L'usage détourné qui en est fait pendant la Révolution est l'objet de la critique des journalistes dans sa part d'exagération. D'abord, l'auteur anonyme de De la puissance des on dit et de leurs dangers en politique et en morale précise que «On dit est un sot dont nous sommes tous les jours la dupe ». Puis le journaliste de Le Nouvelliste de France ${ }^{62}$ ajoute que " on dit est une espèce d'amphybie, moitié démocrate et presque toujours ennemi du bien, de l'ordre et de la monarchie ». Un vrai caméléon donc auquel on peut attacher n'importe quel nom et épithète, aussi exagérés soient-ils.

9. Mettre de la disproportion entre la chose et le mot, joindre aux « conquêtes d'expressions » « les contrastes d'idées », jouer donc sur les discordances. Ainsi nommer « une insurrection populaire » une querelle de quelques gens du peuple.

(59) Dans sa livraison du 30 juillet 1791.

(60) Voir notre étude "L'intrigant dans le discours sectionnaire marseillais (avril-juin 1793) », Dictionnaire des Usages Socio-Politiques 1770-1815, fasc. 4, "Désignants socio-politiques », 2, Paris, Klincksieck, 1989, p. 145-165.

(61) Voir plus largement notre étude, « La "guerre de mots". On dit, nouvelles et dialogues dans la presse révolutionnaire (1791-1793) », dans Michel BIARD, Annie CRÉPIN, Bernard GAINOT (dir.), La plume et le sabre. Mélanges Bertaud, Paris, Publications de la Sorbonne, 2002, p. 101-110.

(62) Dans sa livraison du 27 décembre 1790. 
Le journaliste de Le Lendemain contextualise ce type d'exagération sur le même thème dans une rubrique significativement intitulée «Des insurrections $\|^{63}$. Il considère qu'un ouvrage sur l'histoire de la Révolution se devrait d'avoir « une introduction claire et précise où l'on déterminerait ce que c'est qu'une insurrection, où l'on démontrerait que le seul mouvement populaire qui soit digne de ce nom, le seul qui puisse avoir des suites utiles, est celui d'une nation toute entière, ou du moins de la majorité d'une nation lorsqu'elle est opprimée ». À ce titre, il est possible d'affirmer que « depuis le 14 juillet, il n'y a pas eu une seule insurrection véritable, que tous les mouvements populaires qui ont eu lieu n'étaient que partiels ». Seul donc le 14 juillet est une insurrection : pour le reste ce n'est qu'exagération de la part de journalistes inféodés à un parti, et de clubistes sous les charme des orateurs, précise-t-il également.

Une analyse de même genre se trouve sous la plume du journaliste de la Chronique de Paris dans la rubrique « Des mouvements populaires et de l'opinion publique $»^{64}$. Il y est question des idées légitimes ou non que l'on peut associer à cette expression. Si le mouvement populaire est le fait de l'impulsion d'une multitude usant de la force contre un objet matériel et physique bien connu - on pense de suite à la Bastille - qu'elle détruit, l'expression est légitime par le fait d'une telle association d'idées. Mais s'il s'agit d'une impulsion « guidée par rien », « travaillée d'inquiétudes », ce n'est qu'apparence de mouvement populaire, usage sans fondement d'une telle expression.

Bien sûr, ce type d'exagération se trouve de nouveau dans l'univers des désignants. Ainsi un correspondant de la Chronique de Paris remarque qu'avec « la nouvelle acception du mot enragé », on peut se demander si ce mot « ne serait pas synonyme de philanthrope, d'ami de l'égalité et du peuple, c'est-à-dire à l'opposé en tout ce qu'on appelle aujourd'hui aristocrate $\rangle^{65}$. La disproportion entre la chose et le mot est ici évidente.

Multiplier les citations savantes et les allusions pompeuses constitue la dixième et dernière marche de l'échelle de l'exagération. Nous n'en donnerons pas d'exemple journalistique, puisque Cérutti lui-même use de la citation à propos de la pomme de Newton, tout en ajoutant, « Je m’égare, ou du moins je m'écarte $»^{66}$ !

(63) Dans sa livraison du 20 mars 1791.

(64) Chronique de Paris, 2 mars 1792.

(65) Lambert dans la Chronique de Paris, 19 janvier 1790.

(66) Prospectus, op. cit., p. 34. 
À chaque étape, il s'agit donc bien de « prendre les nouveaux poids de l'exagération ». Cependant, pour mesurer l'ampleur de ce détour par l'exagération parmi les patriotes modérés soucieux d'une langue rationnelle, nous pouvons aussi considérer un courant très particulier parmi les «littérateurs politiques », celui qui a pour but « de prouver aux Aristocrates que tous les rieurs ne sont pas de leur côté, et que l'on peut rire aussi dans le sens de la révolution ». Telle est la formule que l'on retrouve dans La Légende dorée ou Les actes des martyrs pour servir de pendant aux Actes des apôtres, sa visée étant de contrecarrer un des organes les plus importants en 1791 de la presse royaliste, les Actes des apôtres.

Il faut y adjoindre, pour une part, le Dictionnaire national et anecdotique de Pierre-Nicolas Chantreau. Agnès Steuckardt précise à son propos que les libellistes royalistes, en particulier dans Les Actes des apôtres avaient la partie facile, lorsqu'il s'agit de mettre les rieurs de leur côté, puisque "leurs moqueries visent à la fois le discours et sa référence $\aleph^{67}$. Mettre les rieurs du côté des patriotes, en matière d'usage des mots, s'avère plus complexe : cela relève ici conjointement de la fantaisie verbale, du style anecdotique et de l'ironie. Mais là aussi l'exagération tient une bonne place, comme nous l'avons montré en introduisant des exemples extraits du Dictionnaire de Chantreau dans l'échelle de l'exagération proposée par Cérutti. Il en est de même dans La légende dorée, en partant d'un dialogue, qui met en scène les protagonistes de l'exagération, les libellistes royalistes bien connus, Farmian Durosoy et l'abbé Royou.

\section{«Durosoy}

Eh bien! Avez-vous préparé vos batteries contre le nouveau décret? Avez-vous préparé une bonne et prolixe déclamation où vous puissiez, à la faveur des figures de rhétorique, vous dispenser de raisonnement?

Royou

$\mathrm{Oh}$ ! $\mathrm{Du}$ raisonnement, du raisonnement... Fi, laissons cela aux enragés; pour nous, mon ami, déclamons ; surtout, pas de scrupule, entendez-vous; de bons mensonges, bien impudents, bien calomnieux... Dans de si bonnes causes, toutes les armes sont légitimes $»^{68}$.

$\mathrm{Du}$ vocabulaire de l'exagération épinglé dans ce journal, retenons par exemple l'image frappante de «terre de perdition » pour désigner la France dans le vocabulaire du royaliste ce qui fait dire au franc patriote,

(67) Dictionnaire national et anecdotique, p. 55.

(68) La Légende dorée, p. 35. 
«Ah! Cela mériterait explication. Mais pourquoi s'il vous plaît vomir contre ma patrie ce saint torrent de pieuses injures ? $»^{69}$. Si le vocabulaire de l'exagération est ici mis à distance pour prouver " que l'on peut rire aussi dans le sens de la révolution », qu'en est-il alors dans Le Nouveau dictionnaire pour servir à l'intelligence des termes mis en vogue par la Révolution, rédigé par l'abbé Buée - et publié en janvier 1792 -, un royaliste convaincu qui dédie son ouvrage « aux amis de la Révolution, du roi et du sens commun $»^{70}$ ? S'agit-il alors d'exagération au premier degré, ce qui ne serait pas, selon Cérutti, l'exagération proprement dite?

C'est là où il faut s'entendre sur la différence entre la connaissance patriotique, c'est-à-dire sur la base des « véritables principes », et l'exagération et "l'exagération folle et l'exagération hypocrite» (Cérutti) dans lesquelles versent des auteurs comme l'abbé Buée. Il s'agit en effet « d'avertir l'attention» de l'opinion publique sur l'exagération comme "excès de la force ", donc appréhendée dans son principe, et de s'en informer pour donner du génie à langue que l'on utilise. Il s'agit donc bien quelque part de la positiver en la réduisant analytiquement, donc en la régulant. Et Cérutti d'ajouter que celui qui s'intéresse à une telle " langue des effets », " enseigne les diverses manières de troubler, d'agiter les têtes les plus calmes. Il aidera particulièrement les personnages qui parlent en public, et il leur servira en quelque manière de porte-voix pour grossir et propager le son trop faible de leur voix naturelle $\gg^{71}$. L'abbé Buée entre alors dans la catégorie de ceux qui font que « l'exagération est la rhétorique des esprits faibles et la logique des esprits faux $»^{72}$.

Ainsi que 1'a montré Ghislaine Lozachmeur ${ }^{73}$, le discours polémique de Buée se nourrit du langage révolutionnaire lui-même, à distance de toute tentative d'imposer un discours contre-révolutionnaire, distinct du langage patriotique, pour en faire une sorte de crime linguistique. Ainsi, au titre d'une vision naturaliste du rapport entre les mots, les idées et les choses, l'abbé Buée exagère les contrastes entre la chose et le mot, le mot étant une extension indéfinie de la désignation de la chose, ainsi du « ci-devant » défini comme un « Nom qu'on donne à tout ce qui existait,

(69) Ibid., p. 72.

(70) Nous faisons ici référence à l'exemplaire disponible à la Bibliothèque Historique de la Ville de Paris sous la cote BHVP 957104.

(71) Propectus, op. cit., p. 37.

(72) Ibid., p. 35.

(73) Dans sa thèse de doctorat, soutenue à Aix-Marseille I, en 1995 sur Le Nouveau Dictionnaire (A.Q. Buée 1792). Étude d'un discours polémique contre-révolutionnaire. 
et qu'on donnera bientôt à tout ce qui existe $\aleph^{74}$. Contraste qui peut également se faire dans la destruction même de la chose par le mot, ainsi des clubs qui deviennent des "Associations qu'a fait éclore la nouvelle constitution, qui ne veut pas d'associations » par le fait que la Constitution française est la « Désorganisation de tous les pouvoirs ». De même pour l'insurrection : «Q. Qu'est-ce que l'insurrection ? C'est le plus saint des devoirs. En quoi consiste ce devoir ? À piller, à brûler, à massacrer » ${ }^{75}$.

Quant aux termes aériens épinglés par Cérutti, ils entrent ici dans le langage du journaliste, « des folliculaires » : «Quand on sait par cœur les mots despotismes, fanatisme, liberté, aristocratie, poignards, tyrans, etc., on a tout le secret de l'éloquence des folliculaires $»^{76}$. De même l'usage de termes collectifs à forte valeur de généralisation est ici, sans surprise, épinglé à propos du rapport nation-liberté déjà évoqué. À l'entrée « Liberté politique », il est écrit : " C'est la liberté que possède une nation considérée comme un être unique, comme si elle ne formait qu'un seul individu $»^{77}$.

Au Moyen-Âge sous le règne de Charles V (1364-1380), Nicole Oresme traduit l'Éthique d'Aristote : il y associe d'emblée l'expression de " communicacion politique », néologisme qui renvoie à plusieurs termes latins tels que societas, urbanitas, politia, respublica et bien sûr communicatio, à distance des mots « commun », « commune», « communalté». Il franchit donc le pas de la connaissance intuitive, de sens commun, vers la connaissance abstraite, "scientifique ». C'est alors que Nicolas Oresme propose, suite à sa traduction du livre de L'Éthique, une « table des mots divers et étranges » de la science politique, tels que aristocratie, démocratie, civilité, etc., permettant ainsi la genèse et le développement du vocabulaire de cette nouvelle science, du moins dans son expression romane ${ }^{78}$. Il ne s'agit pas là simplement de créer un nouveau lexique politique, dans la mesure où cette table rend compte de l'émergence d'une conscience linguistique du fait politique comme une entité constituée en elle-même qui

(74) Nouveau Dictionnaire pour servir à l'intelligence des termes mis en vogue par la Révolution, op. cit., p. 11.

(75) Ibid, p.14, 15 et 56.

(76) Ibid., p. 53.

(77) Ibid., p. 65.

(78) Nous prenons ici en compte les travaux d'Olivier BERTRAND, en particulier son ouvrage, Du vocabulaire religieux à la théorie politique en France au $14^{\mathrm{e}}$ siècle, les néologismes sous le règne de Charles V (1364-1380), Paris, Connaissances et Savoirs, 2005. 
s'impose progressivement. Nous en avons ailleurs retracé les étapes jusqu'au $\mathrm{XIX}^{\mathrm{e}}$ siècle $^{79}$.

Or nous trouvons, au centre de ce lexique, le mot « extrême » ainsi défini : «Extremes ou extremités sont les bonz des fins, les termes des choses. Mais en especial en matiere moral, extremes sont les habiz et les operacions qui sont hors le moien de vertu en trop et en surhabondance ou en peu et en deffaute. Et pour ce dit l'en que la vertu est moienne et que les vices sont extremes $»{ }^{80}$. Cinq siècles plus tard, la communication politique relève certes d'une forme plus élaborée, mais l'accès à la vertu modératrice, donc moyenne, en matière de langue politique passe aussi par la connaissance des «termes des choses », de leur extrêmes qui sont ainsi visibilisés dans une série d'opérations, liées aux usages, dont le journaliste patriote présente les règles par un travail de réduction analytique. À ses yeux, la langue politique en tant que « langue bien faite » est tout autant une «langue des effets » liée à la force du discours qu'une « langue analytique » issue de la raison.

En fin de compte, nous retrouvons là un phénomène plus général, le fait que l'abord des extrêmes occupe une place centrale au moment où la crise révolutionnaire se traduit par « une surproduction de textes, de prises de parole, de recherches de visibilité dans le champ politique $\gg{ }^{81}$. La part proprement linguistique de l'analyse politique, marquée par une profusion de remarques sur la langue par les journalistes, n'échappe pas à ce processus, bien au contraire.

Jacques Guilhaumou

UMR « Triangle », Université de Lyon, CNRS/ENS-LSH

15 parvis René Descartes BP 7000 - 69342 Lyon cedex 07 guilhaum@newsup.univ-mrs.fr

(79) Voir notre étude, « Les Signes du Politique : Language and Sociability in France from the Fourteenth to the Nineteenth Century», Contributions to the History of Concepts, Volume 4, number 2, 2008, p. 137-159.

(80) Nicole Oresme, « La table des moz divers et estranges », Le livre des Éthiques d'Aristote, Critical Introduction and Notes by Albert Douglas Menut, New York, G.E. Stechert \& Co, 1940, p. 544.

(81) Pierre SERna, La République des girouettes. 1789-1815 et au-delà. Une anomalie politique : la France de l'Extrême centre, Paris, Champ Vallon, 2005, p. 419. 